\title{
Terapia ocupacional com puérperas em enfermaria obstétrica
}

\section{Occupational therapy with puerperas in obstetric ward}

\section{Camylla Varela Luckwü de Oliveira ${ }^{1}$, Alyne Kalyane Câmara de Oliveira ${ }^{2}$}

http://dx.doi.org/10.11606/issn.2238-6149.v30i3p183-188

Oliveira CVL, Oliveira AKCS Terapia ocupacional com puérperas em enfermaria obstétrica. Rev Ter Ocup Univ São Paulo. 2020 set.-dez.;30(3):183-8.

RESUMO: Esta pesquisa objetivou conhecer a opinião de discentes da graduação em Terapia Ocupacional e de profissionais residentes terapeutas ocupacionais sobre a atuação da Terapia Ocupacional junto à puérperas em contexto hospitalar, a partir de suas vivências práticas em enfermaria obstétrica de um hospital universitário da Paraíba. Trata-se de estudo exploratório, descritivo e qualitativo, com 11 discentes de Terapia Ocupacional e 5 residentes terapeutas ocupacionais. Os dados foram coletados por meio de ficha de caracterização do perfil dos participantes e entrevista semiestruturada com o propósito de conhecer a percepção dos participantes sobre a atuação da Terapia Ocupacional com puérperas em enfermaria obstétrica. A partir da Análise de Conteúdo surgiram dois eixos temáticos: "Potencialidades da Terapia Ocupacional com puérperas em enfermaria obstétrica" e "Fragilidades e desafios da Terapia Ocupacional no campo". Resultados revelam contribuições da Terapia Ocupacional para puérperas hospitalizadas ao minimizar impactos causados pelo contexto hospitalar, ajudando-as a ressignificar esse espaço e cotidiano, e a desempenharem o papel ocupacional da maternidade, além de apontarem para a necessidade da inserção de terapeutas ocupacionais e de publicações especí icas no campo.

DESCRITORES: Período pós-parto; Saúde da mulher; Enfermaria; Obstetrícia; Terapia Ocupacional.
Oliveira CVL, Oliveira AKCS Occupational therapy with puerperas in obstetric ward. Rev Ter Ocup Univ São Paulo. 2020 Sept.-Dec.;30(3):183-8.

ABSTRACT: This research aimed to know the opinion of undergraduate students in Occupational Therapy and of resident occupational therapists regarding the performance of Occupational Therapy with puerperal women in a hospital context, from their practical experiences in an obstetric ward of a university hospital in Paraíba. This is an exploratory, descriptive and qualitative study, with 11 Occupational Therapy students and 5 resident occupational therapists. The data was collected through a profile characterization form of the participants and a semi-structured interview. The purpose of this data is to know the participants' perception of the performance regarding Occupational Therapy with puerperal women in an obstetric ward. Two thematic axes emerged from the Content Analysis: "Potentials of Occupational Therapy with Puerperal Women in Obstetric Ward" and "Weaknesses and Challenges of Occupational Therapy in the Field". Results reveal contributions of Occupational Therapy to hospitalized mothers by minimizing impacts caused by the hospital context. This helps them to re-signify their space and daily life, and to play the occupational role of motherhood. Moreover, the results point out the need for the insertion of occupational therapists and specific publications in the field.

KEYWORDS: Postpartum period; Women's health; Nursery; Obstetrics; Occupational Therapy.

Este artigo é parte do Trabalho de Conclusão de Curso de Terapia Ocupacional de Camylla V. L. de Oliveira ${ }^{1}$, sob orientação da Professora Dra. Alyne K. C. de Oliveira ${ }^{2}$, apresentado ao Departamento de Terapia Ocupacional da Universidade Federal

1. Curso de Terapia Ocupacional da Universidade Federal da Paraíba (UFPB), João Pessoa/PB, BR. ORCID: https://orcid.org/ 0000-0003-2875-9425. E-mail: camylla_luckwu@hotmail.com

2. Departamento de Terapia Ocupacional da Universidade Federal da Paraíba (UFPB), João Pessoa/PB, BR. ORCID: https://orcid.org/ 0000-0001-8262-9029. E-mail: alyne.oliveira@academico.ufpb.br

Endereço para correspondência: Departamento de Terapia Ocupacional, Centro de Ciências da Saúde, Universidade Federal da Paraíba Campus I. Cidade Universitária. João Pessoa, PB, Brasil. CEP: 58051-900. E-mail: alyne.oliveira@academico.ufpb.br 


\section{INTRODUÇÃO}

$\mathrm{O}^{\mathrm{s}}$ puerpério, apesar de variável e impreciso, pode ser entendido como a etapa de manifestações involutivas, ao estado pré-gravídico, das transformações locais e sistêmicas provocadas pela gravidez e parto, ao mesmo tempo em que nele deve ocorrer o exercício da maternidade $^{1}$. Zanatta e Pereira ${ }^{2}$ apontam que durante a gestação a mulher já passa a ser vista e a se enxergar de modo diferente, passando do papel ocupacional de filha e mulher, para o papel de mãe.

Papéis ocupacionais organizam o comportamento e o uso do tempo, conduzem as expectativas sociais a realização, envolvem os indivíduos em uma estrutura social e colaboram para a identidade pessoal ${ }^{3}$. O papel ocupacional materno inclui os cuidados com o recém-nascido $(\mathrm{RN})$ e o cotidiano da mãe deve se adaptar à rotina do bebê.

Behar $^{4}$ revela que essa mudança repentina no cotidiano da puérpera, a afastando de interesses pessoais, e a dificuldade de conciliação dos papéis ocupacionais, são fatores que podem produzir estresse, cansaço, frustrações e provocar sensação de sobrecarga.

Isso se intensifica no caso de mães com bebê que, ao nascer, precisa de cuidados em Unidade de Terapia Intensiva Neonatal (UTIN) ou em Unidade de Cuidado Intermediário Neonatal (UCIN); puérperas com bebê em alojamento conjunto na maternidade; ou que enfrentam complicações no pós-parto e que demoram a receber alta. Além dos fatores estressantes habituais dos primeiros dias de adaptação ao/do bebê, no hospital a puérpera não possui a comodidade do lar, o amparo da família/amigos e necessita lidar com a rotina hospitalar. O período de hospitalização pode representar desamparo para a mulher e familiares ${ }^{5}$.

As puérperas em contexto hospitalar se tornam coadjuvantes no cuidado do bebê, onde o toque mãe-bebê muitas vezes é sob autorização profissional e nos horários possíveis entre os atendimentos, o que as faz se sentirem culpadas por achar que não sabem cuidar do próprio filho e se colocarem numa posição periférica de apoio ${ }^{6}$.

A Terapia Ocupacional é uma profissão que pode auxiliar puérperas a vivenciarem uma rotina saudável no contexto hospitalar e proporcionar bem-estar por meio de diferentes técnicas, métodos e abordagens ${ }^{7,8}$. De Carlo e Luzo $^{5}$ referem que, independente do público em questão, existem algumas ações principais desenvolvidas pelo terapeuta ocupacional no contexto hospitalar, como: intervenção no cotidiano hospitalar com objetivo de promover qualidade de vida durante a internação do sujeito; ajudar a família no processo terapêutico; preparar para alta; avaliar desempenho em atividades de vida diária e intervir quando necessário; atuar no cotidiano do paciente; proporcionar momentos de expressar temores e percepções sobre o problema (motivo da internação); estimular a criatividade e o lazer; minimizar o impacto da hospitalização; prevenir incapacidades; dentre outros.

A atenção a puérperas em contexto hospitalar tem sido desenvolvida no cenário da enfermaria obstétrica do Hospital Universitário Lauro Wanderley (HULW), em João Pessoa/PB, Brasil, por profissionais residentes terapeutas ocupacionais e por discentes do Curso de Terapia Ocupacional da Universidade Federal da Paraíba (UFPB) em disciplina teórico-prática da graduação. A disciplina “Áreas de intervenção da Terapia Ocupacional e cenários de prática III" promove o estudo, a observação e a experimentação do processo da Terapia Ocupacional em cenários hospitalares, tendo como referencial as políticas de saúde local e nacional ${ }^{9}$. Compõe a matriz curricular obrigatória ofertada pelo departamento de Terapia Ocupacional da UFPB para discentes do sexto período do curso. Os residentes terapeutas ocupacionais trabalham com puérperas nesse cenário dentro de programas de residência da área, oferecidos pela UFPB, a saber: Residência Integrada Multiprofissional em Saúde Hospitalar e Residência Multiprofissional em Saúde Mental.

Desta maneira, o presente estudo buscou conhecer a opinião destes discentes da Terapia Ocupacional e profissionais residentes terapeutas ocupacionais sobre a atuação da Terapia Ocupacional junto à puérperas em contexto hospitalar, a partir de suas vivências práticas em enfermaria obstétrica de um hospital universitário da Paraíba, e se justifica pela relevância de levantar dados acerca desse campo de atuação para profissão, por meio da escuta dos que tem experimentado a construção da Terapia Ocupacional na assistência à mulher no puerpério.

\section{PROCEDIMENTOS METODOLÓGICOS}

Trata-se de um estudo exploratório, descritivo, com abordagem qualitativa, que teve como participantes discentes do sexto período de graduação em Terapia Ocupacional que cursaram a disciplina teórico-prática "Áreas de intervenção da Terapia Ocupacional e cenários de prática III" ofertada pelo departamento de Terapia Ocupacional da UFPB e profissionais residentes terapeutas ocupacionais, que vivenciaram práticas da Terapia Ocupacional com puérperas em enfermaria obstétrica do Hospital Universitário Lauro Wanderley (HULW), em João Pessoa/PB, Brasil.

A escolha dos participantes se justifica pelo fato de os discentes terem vivenciado a prática com puérperas em enfermaria obstétrica. No caso dos profissionais residentes se justifica por sua atuação no cenário do estudo. 
Oliveira CVL, et al. Terapia ocupacional com puérperas em enfermaria obstétrica. Rev Ter Ocup Univ São Paulo. 2020 set./dez.;30(3):183-8.

Os discentes participantes vivenciaram a prática na unidade materno-infantil do hospital 1 ou 2 vezes por semana. Os residentes tiveram atuação 5 vezes por semana, ao longo de um período entre 2 e 5 meses no cenário do estudo. Foram realizados contatos com os 12 discentes que cursaram a disciplina no período letivo 2018.1 e com 6 residentes que ingressaram nos programas em 2017 e 2018: 4 da residência em saúde hospitalar com ênfase na criança/adolescente e 2 da residência em saúde mental. Aceitaram participar 11 discentes e 5 residentes, com o total de 16 participantes.

A aplicação dos instrumentos de coleta de dados foi desenvolvida no departamento de Terapia Ocupacional, em sala reservada para este fim. Para os discentes e residentes foram agendados horários prévios, respeitando a rotina do local e as suas atividades acadêmicas.

A coleta de dados ocorreu durante o mês de fevereiro de 2019. Os entrevistados responderam a uma ficha para caracterização do perfil dos participantes e responderam a entrevista semiestruturada com o propósito de conhecer a percepção dos participantes sobre a atuação da Terapia Ocupacional com puérperas em enfermaria obstétrica, a partir de oito questões. Os instrumentos foram elaborados pela equipe pesquisadora, sendo a entrevista gravada em áudio e transcrita na íntegra.

Os dados da ficha de caracterização foram analisados descritivamente e os dados qualitativos resultantes das entrevistas, de acordo com a técnica de Análise de Conteúdo na modalidade temática ${ }^{10}$. Na primeira etapa de análise foi feita leitura detalhada e repetida das transcrições, para apreensão das ideias centrais. Na segunda etapa, leitura transversal de cada questão para identificar os dados mais relevantes que construíram os eixos temáticos.

O estudo foi aprovado pelo Comitê de Ética em Pesquisa em Seres Humanos, conforme diretrizes da Resolução 466/2012 da Comissão Nacional de Ética em Pesquisa (CAAE 03099618.5.0000.8069) e os participantes assinaram o Termo de Consentimento Livre e Esclarecido.

\section{RESULTADOS E DISCUSSÃO}

Dados de caracterização revelam o perfil dos participantes, que tinham entre 20 e 27 anos, sendo 12 do sexo feminino e 4 do sexo masculino. Destes, 3 dos residentes tinham experiência prévia no campo com puérperas, por meio da disciplina em contexto hospitalar na graduação, e nenhum dos participantes possuía capacitação prévia no campo.

A partir da análise das entrevistas foram definidos dois eixos temáticos: "Potencialidades da Terapia Ocupacional com puérperas em enfermaria obstétrica" e "Fragilidades e desafios da Terapia Ocupacional no campo". Trechos das entrevistas são apresentados, de forma que a letra "RM" faz referência a um residente em saúde mental, "RH" a um residente em saúde hospitalar e "D", a um discente. Os números identificam a ordem de ocorrência das entrevistas.

\section{Potencialidades da Terapia Ocupacional com puérperas em enfermaria obstétrica}

Nesse eixo, apresentam-se percepções dos participantes sobre as intervenções com puérperas em enfermaria obstétrica, com destaque para as potencialidades da profissão na atuação com este público.

No que se refere à intervenção da Terapia Ocupacional houve unanimidade entre os participantes sobre a importância das intervenções. Segundo eles, a importância está relacionada ao fato da profissão analisar e intervir no cotidiano, na rotina, nas diversas ocupações humanas, na relação com os papéis ocupacionais. No caso da maternidade, além de preocupar-se com os impactos trazidos pelo contexto da hospitalização, pode contribuir na ressignificação do espaço hospitalar e das situações em que cada mulher se encontra.

\footnotetext{
"Eu considero as práticas da TO muito importantes (...) porque lá na obstetrícia as mulheres ficam muito esquecidas... seu corpo, o lado pessoal, o contexto social e ficam ali ociosas, muitas vezes, perdem seus papéis ocupacionais e é muito importante a TO para trabalhar essa questão (...). Então a gente está ressignificando aquele ambiente para ela... o TO tem o diferencial de conseguir enxergar para além da condição física" (RH2).

"De extrema importância, uma vez que os papéis ocupacionais... nesse período de hospitalização são rompidos... as mulheres costumam apresentar muita angústia, muita ansiedade... às vezes elas passam um periodo muito longo para acompanhar o filho... isso gera sofrimento de qualquer maneira... então a gente tenta ressignificar esse espaço de alguma maneira" (RM4).
}

As entrevistas apontam para a importância do acompanhamento profissional que possa ajudar as puérperas a enfrentar essa fase com menos impacto no desempenho e na estrutura do seu cotidiano e de suas ocupações, minimizando as repercussões ocasionadas pelo contexto de hospitalização.

Nessa direção, é inegável a contribuição da Terapia Ocupacional, que atua com o propósito de melhorar ou possibilitar a participação em papéis, hábitos e rotinas. Segundo Crepeau e Schell11 ${ }^{11}$ a Terapia Ocupacional "volta-se para tais papéis tendo como foco as ocupações e o desempenho, buscando compreender o desempenho 
Oliveira CVL, et al. Terapia ocupacional com puérperas em enfermaria obstétrica. Rev Ter Ocup Univ São Paulo. 2020 set./dez;;30(3):183-8.

dos papéis ocupacionais pelo indivíduo e a repercussão do desempenho na vida cotidiana" 12 .

O terapeuta ocupacional atua com mulheres no puerpério mediante as condições de vulnerabilidade a qual estão submetidas, como o exercício de um novo papel ocupacional, o da maternidade, que pode gerar processos de ansiedade, estresse e angústia, principalmente na hospitalização, seja em decorrência da dinâmica hospitalar, da situação/problema que a faz necessitar de cuidados especializados, bem como do que o ambiente hospitalar a remete ${ }^{13}$.

Pergher et al. ${ }^{14}$ apontam que a permanência hospitalar e a privação de contato com o bebê em internamento aumentam o estresse materno, e que a mãe que comumente enfrenta frustrações e inseguranças devido a situações atípicas, como prematuridade do bebê ou complicações pós-parto, necessita superá-las para relacionar-se com o filho e esse contexto pode fragilizar a relação inicial entre mãe-bebê, o que demanda atenção da equipe.

O terapeuta ocupacional é um dos profissionais que pode compor a equipe hospitalar, mas essa incorporação ainda não está consolidada ${ }^{15}$. A especialidade do terapeuta ocupacional em contexto hospitalar "visa contribuir com a proteção, promoção, prevenção, recuperação da saúde e reabilitação do indivíduo e da coletividade, pautado na concepção de integralidade e humanização da assistência à saúde ${ }^{5,16}$ ", tendo assim, muito a contribuir na atenção à puérperas em contexto de internação.

\section{Fragilidades e desafios da Terapia Ocupacional no campo}

No que concerne às fragilidades da Terapia Ocupacional na prática vivenciada com puérperas, os participantes descreveram a falta de inserção do profissional terapeuta ocupacional na enfermaria obstétrica, o desconhecimento da profissão entre os demais profissionais e o distanciamento da possibilidade do trabalho multiprofissional e/ou interprofissional. Além de esta ausência favorecer uma prática fragmentada, uma vez que, as intervenções não acontecem continuamente, mas como ações pontuais a cada semestre, quando se tem a oferta da prática da disciplina na enfermaria obstétrica e por alguns meses no ano, quando se tem residentes terapeutas ocupacionais atuando na unidade.

“como fragilidade... tinha a questão da ausência do profissional de referência naquele setor" (RH1).

"também vejo como fragilidade porque alguns profissionais não têm a visão ainda do que o TO pode fazer ali dentro do hospital" (D2). "a fragilidade ainda é da questão da multidisciplinaridade naqueles espaços e também de reconhecimento da própria Terapia Ocupacional" (D8).

Tais fragilidades sugerem a necessidade da prática profissional permanente da Terapia Ocupacional neste espaço, mais contato e interlocução com os profissionais da equipe, e a contratação de terapeutas ocupacionais para a enfermaria obstétrica do hospital.

A inserção do profissional terapeuta ocupacional nesse contexto é assegurada pela Resolução COFFITO $n^{\circ} 418 / 2012$, que estabeleceu e fixou os parâmetros assistenciais terapêticos ocupacionais nas diversas modalidades de atendimento, incluindo em Contextos Hospitalares, bem como, pela Resolução COFFITO n ${ }^{\circ}$ 429/2013, que reconheceu e disciplinou a especialidade de Terapia Ocupacional em Contextos Hospitalares ${ }^{16-18}$.

Contudo, apesar da garantia legal, a quantidade de terapeutas ocupacionais contratados por tais serviços ainda se mostra insuficiente e nem sempre consegue atender à todas unidades hospitalares. Isto pode ser observado no próprio HULW, em que mesmo com a contratação de novos profissionais, os terapeutas ocupacionais que existem não alcançam todas as demandas do hospital geral.

Ao mesmo tempo em que se entende a necessidade do profissional terapeuta ocupacional de referência no serviço em benefício da assistência prestada às puérperas, reforça-se a relevância da graduação e pós-graduação nos movimentos de expansão de campos de atuação para a Terapia Ocupacional, possibilitando a formação e o aperfeiçoamento das competências e habilidades profissionais no campo de conhecimento e prática profissional, com destaque para as residências multiprofissionais em saúde.

Na percepção dos participantes, destacou-se como fragilidade no exercício da profissão a escassez de literatura em relação a especificidade da Terapia Ocupacional com puérperas.

"é um campo que a gente está ganhando ainda referencial teórico, na verdade a gente está começando a abranger ainda (...) com a puérpera é um campo que está sendo conquistado agora" RH2).

"tem escassez muito grande na literatura sobre o trabalho da Terapia Ocupacional com as puérperas... eu busquei outros livros, procurei na biblioteca do próprio hospital. Eu fui ler sobre o pós-parto, porque você também não encontra, o que você encontra é cuidados no pós-parto" (RH3).

Segundo Galheigo ${ }^{15}$, a produção sobre as intervenções da Terapia Ocupacional no hospital com puérperas e neonatos, 
Oliveira CVL, et al. Terapia ocupacional com puérperas em enfermaria obstétrica. Rev Ter Ocup Univ São Paulo. 2020 set./dez.;30(3):183-8.

assim como com gestantes, retrata o movimento de cuidar da saúde da mulher e do recém-nato precocemente, exercendo um fator adicional de proteção à saúde materno-infantil. A partir de seu estudo da produção científica brasileira de 1990 a 2006, encontrou relatos de experiência que registravam a participação do terapeuta ocupacional em iniciativas compartilhadas com equipe multiprofissional de saúde, com início no hospital e continuidade em ambulatórios de seguimento.

As recomendações relativas à assistência obstétrica e neonatal, idealizadas pela Organização Mundial de Saúde (OMS) parecem também impactar nas produções, ainda que incipientes, sobre as práticas desenvolvidas por terapeutas ocupacionais junto a esta população, seja nas atividades de promoção, proteção e apoio ao aleitamento materno ${ }^{19}$; seja no acompanhamento e orientação à puérpera e família do neonato pós-alta em UTIN, UCIN e em acompanhamento ambulatorial ${ }^{7,8,20}$, seja no olhar sobre o desempenho ocupacional pós-cirurgia em mulheres submetidas à cesárea ${ }^{21}$ (no puerpério imediato), que fomenta as discussões sobre protagonismo da mulher e práticas baseadas em evidências frente ao movimento e políticas de humanização ao parto e nascimento, os quais priorizam bem-estar da mãe e do bebê ${ }^{22}$.

\section{CONSIDERAÇÕES FINAIS}

Ao levar em consideração a percepção de discentes de graduação de Terapia Ocupacional e profissionais residentes terapeutas ocupacionais em relação a atuação da profissão com puérperas em enfermaria obstétrica, a partir de práticas desenvolvidas em um hospital universitário, e destacar contribuições desta atuação, este estudo traz subsídios para embasar as práticas da profissão no contexto hospitalar, além da problematização acerca da inserção do terapeuta ocupacional neste campo de atuação e das produções científicas.

Para os participantes a importância da prática da Terapia Ocupacional com as puérperas se justifica por ser, o terapeuta ocupacional, um profissional que analisa e intervêm no cotidiano, na rotina, nas ocupações, na relação com os papéis ocupacionais, aspectos estes, comumente impactados na vida da mulher-mãe no período do puerpério. Além de preocupar-se com as repercussões do processo de hospitalização para a puérpera, para o desempenho da sua maternidade e para a díade mãe-bebê, contribui na ressignificação do espaço hospitalar e das situaçõesproblemas, causas da internação.

Fragilidades e desafios foram identificados, como a necessidade de mais profissionais terapeutas ocupacionais em trabalho com puérperas e mais produção científica específica da Terapia Ocupacional na temática, para melhor fundamentação das ações. Para atestar este último, faz-se necessária produção de revisão sistemática da literatura.

Por fim, mediante experiência local, indica-se a inserção do profissional terapeuta ocupacional no cenário da obstetrícia do HULW, como também em demais unidades hospitalares materno-infantil distribuídas pelo estado da Paraíba, em que se conhece a realidade de não ter também este profissional na equipe, como forma de possibilitar mais campo de estudo para as graduações e pós-graduações em Terapia Ocupacional, abertura de estágios, bem como um atendimento mais direcionado, especializado e contínuo para a população de mulheres puérperas que necessitam desses cuidados.

Contribuição dos autores: Camylla V. L. de Oliveira foi responsável pelas etapas de coleta dos dados, análise e redação preliminar do texto; Alyne K. C. de Oliveira participou da concepção e delineamento do estudo, orientação e redação final do texto.

\section{REFERÊNCIAS}

1. Vieira F, Bachion MM, Salge AKM, Munari DB. Diagnósticos de enfermagem da NANDA no período pós-parto imediato e tardio. Esc Anna Nery. 2010;14(1):83-9. doi: http://dx.doi. org/10.1590/S1414-81452010000100013.

2. Zanatta E, Pereira CRR. "Ela enxerga em ti o mundo": a experiência da maternidade pela primeira vez. Temas Psicol. 2015;23(4):959-72. doi: http://dx.doi.org/10.9788/TP2015.4-12.

3. Cordeiro JR. Validação da lista de identificação de papéis ocupacionais em pacientes portadores de doença pulmonar obstrutiva crônica (DPOC) no Brasil [tese]. São Paulo: Universidade Federal de São Paulo - USP; 2005. Disponível em: http://repositorio.unifesp.br/handle/11600/20599.

4. Behar RCR. A maternidade e seu impacto nos papéis ocupacionais de primíparas [monografia]. Paraíba: Universidade Federal da Paraíba - UFPB; 2018. Disponível em: https://repositorio.ufpb.br/ jspui/handle/123456789/2384.pdf.

5. De Carlo MMRP, Luzo MCM. Terapia ocupacional: reabilitação física e contextos hospitalares. São Paulo: Rocca; 2018. 
Oliveira CVL, et al. Terapia ocupacional com puérperas em enfermaria obstétrica. Rev Ter Ocup Univ São Paulo. 2020 set./dez;;30(3):183-8.

6. El-Aouar IS. Tornando-se mãe de um bebê prematuro: a emergência de significados de maternidade a partir da experiência de cuidado na unidade de terapia intensiva neonatal e ambulatório do método mãe canguru [dissertação]. Bahia: Universidade Federal da Bahia - UFBA; 2016. Disponível em: https://pospsi. ufba.br/sites/pospsi.ufba.br/files/isadora_sebadelhe.pdf.

7. Dittz ES, Melo DCC, Pinheiro ZMM. A terapia ocupacional no contexto da assistência à mãe e à família de recém-nascidos internados em unidade de terapia intensiva. Rev Ter Ocup Univ São Paulo. 2006;17(1):42-7. doi: https://doi.org/ 10.11606/issn.2238-6149.v17i1p42-47.

8. Alves CO, Rodrigues RP, Dittz ES. Oficina de culinária: resgate da cotidianidade das mães acompanhantes de recémnascidos de uma unidade de terapia intensiva neonatal. Rev Min Enferm. 2008; 12(1):127-30. doi: http:/www.dx.doi.org/ S1415-27622008000100018.

9. Universidade Federal da Paraíba. Centro de Ciências da Saúde. Projeto Pedagógico do Curso de Terapia Ocupacional. João Pessoa; 2008. Disponível em: http://www.ccs.ufpb.br/coordto/ contents/documentos/ppc/ppc_to_2008_aprovado_consepealteracoes-compressed.pdf

10. Bardin L. Análise de conteúdo. 7a ed. São Paulo: LDA/Almeidina Brasil; 2016.

11. Crepeau EB, Cohn ES, Schell BAB. Analisando ocupações e atividades. In: Crepeau EB, Cohn ES, Schell BAB. Willard \& Spackman: Terapia Ocupacional. 11a ed. Rio de Janeiro: Guanabara Koogan; 2011.

12. Salles MM, Matsukura TS. Estudo de revisão sistemática sobre o uso do conceito de cotidiano no campo da terapia ocupacional no Brasil. Cad Ter Ocup Ufscar. 2013;21(2):26573. doi: https://doi.org/10.4322/cto.2013.028.

13. Camacho RS, Cantinelli FS, Ribeiro CS, Cantilino A, Gonsales BK, Braguittoni E. Transtornos psiquiátricos na gestação e no puerpério: classificação, diagnóstico e tratamento. Rev Psiquiatr Clín. 2006;33(2):92-102. doi: http://dx.doi.org/10.1590/S0101-60832006000200009.

14. Pergher DNQ, Cardoso CL, Jacob AV. Nascimento e internação do bebê prematuro na vivência da mãe. Estilos Clin. 2014;19(1):40-56. doi: https://doi.org/10.11606/ issn.1981-624.v19i1p40-56.

15. Galheigo SM. Domínios e temáticas no campo das práticas hospitalares em terapia ocupacional: uma revisão da literatura brasileira de 1990 a 2006. Rev Ter Ocup Univ São Paulo. 2007;18(3):113-21. doi: https:// doi.org/10.11606/issn.2238-6149.v18i3p113-12.

16. Dahdah DF, Frizzo HCF, Fangel LMV. Terapia ocupacional em contextos hospitalares-caracterização do ensino nos cursos de graduação universitários brasileiros. Rev Ter Ocup Univ São Paulo. 2014;25(1):70-9. doi: http://dx.doi.org/10.11606/ issn.2238-6149.v25ilp70-9.

17. Conselho Federal de Fisioterapia e Terapia Ocupacional. Fixa e estabelece os Parâmetros Assistenciais Terapêuticos Ocupacionais nas diversas modalidades prestadas pelo Terapeuta Ocupacional e dá outras providências. Resolução COFFITO no 418 de 04 de junho de 2012. Diário Oficial da União no 109, Seção 1, página 227 de 06/06/2012, Poder Executivo, Brasília, DF; 2012. Disponível em: http://www. coffito.org.br/publicacoes/pub_view.asp? cod $=2279 \&$ psecao $=9$.

18. Conselho Federal de Fisioterapia e Terapia Ocupacional. Reconhece e disciplina a especialidade de Terapia Ocupacional em Contextos Hospitalares, define as áreas de atuação e as competências do terapeuta ocupacional especialista em Contextos Hospitalares e dá outras providências. Resolução COFFITO no 429 de 08 de julho de 2013. Diário Oficial da União $n^{\circ} 169$, Seção 1, página 116 de 02/09/2013, Poder Executivo, Brasília, DF; 2013. Disponível em: http://www.coffito.org.br/publicacoes/pub_ view.asp? $\operatorname{cod}=2495 \&$ psecao $=9$.

19. Pitonyak JS. The issue is occupational therapy and breastfeeding promotion: our role in societal health. Am J Occup Ther. 2014;68:90-6. doi: http://dx.doi.org/10.5014/ ajot.2014.009746.

20. Menegat D. Mãe-bebê de risco: os desafios da interação inicial no contexto de internação hospitalar [dissertação]. São Carlos: Programa de Pós-graduação em Terapia Ocupacional do Centro de Ciências Biológicas e da Saúde da Universidade Federal de São Carlos - UFSCar; 2016. Disponível em: https://repositorio.ufscar.br/handle/ ufscar/7939.

21. Medeiros TML, Marcelino JFQ. Percepção de puérperas sobre o seu desempenho ocupacional no pós-operatório da cesariana. Cad Bras Ter Ocup. 2018;26(1):97-109. doi: http://dx.doi.org/10.4322/2526-8910.ctoAO0960.

22. Leal MC. Parto e nascimento no Brasil: um cenário em processo de mudança. Cad Saúde Pública. 2018;34(5):1-3. doi: 10.1590/0102-311X00063818. 